19

\title{
Люминесценция двухчастичного комплекса из сферической квантовой точки и плазмонной наноглобулы во внешнем магнитном поле
}

\author{
(C) М.Г. Кучеренко, В.М. Налбандян
}

Центр лазерной и информационной биофизики, Оренбургский государственный университет, 460018 Оренбург, Россия

e-mail: clibph@yandex.ru

Поступила в Редакцию 18.05.2020 г.

В окончательной редакции 18.05.2020 г.

Принята к публикации 07.07.2020 г.

На основе специально созданной теоретической модели произведены расчеты частотных зависимостей интенсивности свечения двухкомпонентной системы „экситон-активированная полупроводниковая квантовая точка (KT, QD) - плазмонная наночастица (НЧ, NP)“ в постоянном магнитном поле. В отличие от предыдущих моделей произведен выход за рамки приближения дипольной поляризуемости сферической НЧ. При расчете индуцированного дипольного момента НЧ учитывался неоднородный характер поля, создаваемого содержащей экситон КТ. Показано, что с изменением индукции внешнего магнитного поля наблюдается трансформация спектров экситонной люминесценции такой системы в результате экситон-плазмонного взаимодействия между частицами кластера и замагниченности электронной плазмы НЧ. Произведен учет конкуренции радиационных и безызлучательных каналов распада возбужденного состояния двухчастичного комплекса. Показано, что в спектрах скорости безызлучательного переноса энергии от КТ к НЧ, а также спонтанного испускания нанокомплекса кроме дипольных полос образуются полосы мультипольных переходов высших порядков, расщепляющиеся на дублетные компоненты в магнитом поле.

Ключевые слова: сферическая квантовая точка, замагниченная плазмонная наночастица, люминесценция двухчастичного комплекса, мультипольные поляризуемости.

DOI: $10.21883 /$ OS.2020.11.50184.153-20

\section{Введение}

Типичными объектами современной наноэлектроники и фотоники являются полупроводниковые и металлические наночастицы (НЧ, NP) разной формы, в которых под действием электромагнитного излучения генерируются коллективные электронные возбуждения в виде экситонов и плазмонов [1,2]. Взаимодействие экситонов и плазмонов друг с другом вызывает существенный интерес благодаря его определяющей роли в поверхностно-усиленной рамановской спектроскопии (SERS) [3], экситонной люминесценции гибридных НЧ вблизи металлических поверхностей и их влиянии на реакции гетерогенного катализа [4], в увеличении КПД ячеек солнечных элементов [5], а также во многих других процессах в различных областях наноэлектроники. В связи с этим для более эффективного и прецизионного контроля технологических процессов и работы электронных устройств нового поколения становится важным развитие методов оперативного управления интенсивностью экситон-плазмонного взаимодействия в наноструктурах с помощью внешнего магнитного поля.

Экситоны, генерируемые в квантовых точках (KT, QD) или молекулярных $J$-агрегатах, могут образовывать сильные или слабые связи с плазмонами в зависимости от структуры наносистемы и ее энергетических харак- теристик [6-8], что приводит к различным уникальным оптическим свойствам. Металлические НЧ и планарные структуры с плазмонным слоем могут существенно изменять радиационные характеристики близлежащих КТ в результате экситон-плазмонного взаимодействия. В работах [9-11] экспериментально исследованы особенности люминесценции КТ в ближнем поле металлических НЧ. Наблюдалось увеличение поглощения КТ в 4 раза и возрастание интенсивности флуоресценции почти на порядок. В ряде работ исследовано экситонплазмонное взаимодействие в композитных проводящих частицах, покрытых слоем $J$-агрегатов [12], а также в планарной системе „металлическая пленка-слой молекулярных $J$-агрегатов“ $[13-15]$. Так, в работе [15] численно реализована математическая модель и показано, что поверхностные плазмоны в металлической пленке значительно увеличивают скорость безызлучательного переноса энергии экситонов Френкеля из одного монослоя $J$ - агрегатов в другой.

Оказалось, что эффективность плазмонного влияния на экситонные процессы зависит не только от геометрической конфигурации системы и состояния электронного газа в металле [16], но также и от внешнего магнитного поля, способного изменять поляризационные характеристики проводящей частицы [17-21]. Так, например, в [21] обнаружено влияние магнитного поля 


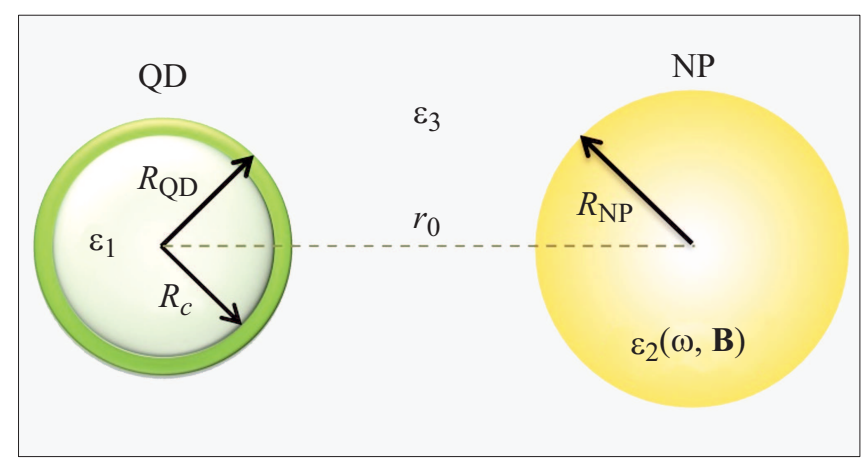

Рис. 1. Геометрическая конфигурация исследуемой системы.

на экситонную люминесценцию и случайную генерацию в ближнем УФ диапазоне пленок $\mathrm{ZnO}$, сформированных из хаотично ориентированных наностержней, покрытых НЧ серебра при комнатной температуре. При наличии магнитного поля в такой системе наблюдалось ярко выраженное усиление люминесценции, тогда как в отсутствие НЧ серебра эффект магнитополевого усиления свечения не наблюдался.

В настоящей работе предложена теоретическая модель электронных процессов в композитной системе КТ-НЧ, в рамках которой исследованы механизм и эффективность магнитной модуляции радиационных и безызлучательных процессов в системе с учетом неоднородности ближнего поля и анизотропных характеристик мультипольных поляризуемостей замагниченной НЧ.

\section{Математическая модель}

Рассмотрим две сферические НЧ (рис. 1), одна из которых представляет собой экситон-активированную полупроводниковую сферическую КТ радиуса $R_{\mathrm{QD}}=R_{c}+\Delta$ содержащейся в ее ядре $r^{\prime}<R_{c}$ электрондырочной парой или экситоном Ванье-Мотта, а другая - металлическую НЧ радиуса $R_{\mathrm{NP}}$ с расстоянием $r_{0}>R_{\mathrm{QD}}+R_{\mathrm{NP}}$ между центрами частиц.

Поле активированной КТ осциллирует со временем $t$ на частоте $\omega$ дипольного источника с моментом $\mathbf{p}=\mathbf{p}_{0} \exp (-i \omega t)$. Это поле наводит в НЧ дипольный момент $\mathbf{p}_{2}$. Полагаем, что диэлектрическая проницаемость КТ $\varepsilon_{1}$ не обладает выраженной частотной дисперсией, тогда как диэлектрическая проницаемость $\varepsilon_{2}(\omega)$ проводящей НЧ, наоборот, имеет типичную для металлов сильную дисперсию в области частот $\omega$ экситонного перехода. Во внешнем магнитном поле индукции В электронная плазма металла приобретает анизотропные свойства, и диэлектрическая проницаемость $\varepsilon_{2}$ становится тензором $\overleftrightarrow{\varepsilon}_{2}(\omega \mid \mathbf{B})$ второго ранга [22]. Прозрачная среда характеризуется диэлектрической постоянной $\varepsilon_{3}$.

В приближении холодной замагниченной электронной плазмы тензор $\overleftrightarrow{\varepsilon}_{2}(\omega \mid \mathbf{B})$ диэлектрической проницаемости в декартовой системе координат записывается в виде [23]

$$
\overleftrightarrow{\varepsilon}_{2}(\omega \mid \mathbf{B})=\left(\begin{array}{ccc}
\varepsilon_{\perp}(\omega \mid \mathbf{B}) & i g(\omega \mid \mathbf{B}) & 0 \\
-i g(\omega \mid \mathbf{B}) & \varepsilon_{\perp}(\omega \mid \mathbf{B}) & 0 \\
0 & 0 & \varepsilon_{\|}(\omega)
\end{array}\right)
$$

где $\quad \varepsilon_{\perp}(\omega \mid \mathbf{B})=1-\frac{\omega_{p}^{2} K}{\omega\left(K^{2}-\Omega_{L}^{2}\right)} \quad \varepsilon_{\|}(\omega)=1-\frac{\omega_{p}^{2}}{\omega K}$, $g(\omega \mid \mathbf{B})=\frac{\omega_{p}^{2} \Omega_{L}}{\omega\left(K^{2}-\Omega_{L}^{2}\right)}, \quad K=(\omega+i \gamma), \quad \gamma \quad$ - частота электронных столкновений (коэффициент диссипации), $g(\omega \mid \mathbf{B})$ - модуль вектора гирации, $\omega_{p}=\sqrt{4 \pi e^{2} n_{e} / m}-$ ленгмюровская (плазменная) частота, $\Omega_{L}=e|\mathbf{B}| / m c-$ ларморовская (циклотронная) частота электрона в магнитном поле индукции $B$.

На малых расстояниях $r_{0}$ от КТ и/или сравнительно больших радиусах $R_{\mathrm{NP}} \sim r_{0}$ НЧ поле $E(\omega)$ диполя р уже нельзя считать однородным, и часто используемое для описания отклика на это поле электрической дипольной поляризуемости $\alpha(\omega)$ НЧ [16] становится некорректным. Ниже мы развиваем более общую модель, которая учитывает неоднородность поля $\mathbf{E}(\omega, \mathbf{r})$ как внутри НЧ, так и снаружи нее, а также анизотропию замагниченной электронной плазмы НЧ.

Потенциалы $\varphi_{j}(r, \theta)$ электрического поля внутри НЧ $\left(j=2, r<R_{\mathrm{NP}}\right)$ с тензором $\overleftrightarrow{\varepsilon}_{2}(\omega \mid \mathbf{B})$ диэлектрической проницаемости ее замагниченной плазмы и снаружи $\left(j=3, r>R_{\mathrm{NP}}\right)$ - в среде с диэлектрической проницаемостью $\varepsilon_{3}$, создаваемого точечным зарядом $q$, находящимся в точке $\left(r_{0}, 0, r_{0}>R_{\mathrm{NP}}\right)$, вне шара могут быть записаны в виде

$$
\begin{aligned}
& \varphi_{2}\left(r, \theta ; r_{0} \mid \mathbf{B}\right)=q \sum_{l=1}^{\infty}(2 l+1) \mathbf{r}_{0} \varepsilon_{l}^{-1}(\omega \mid \mathbf{B}) \mathbf{r} \frac{r^{l-1}}{r_{0}^{l+2}} P_{l}(\cos \theta) \\
& r<R_{\mathrm{NP}} \\
& \varphi_{3}\left(r, \theta ; r_{0} \mid \mathbf{B}\right)=\frac{q}{\varepsilon_{3} R_{A}}+\frac{q}{\varepsilon_{3}} \sum_{l=1}^{\infty} l \mathbf{r}_{0}\left[\varepsilon_{3}-\overleftrightarrow{\varepsilon}_{2}(\omega \mid \mathbf{B})\right] \\
& \quad \times \overleftrightarrow{\varepsilon}_{l}^{-1}(\omega \mid \mathbf{B}) \mathbf{r} \frac{R_{\mathrm{NP}}^{2 l+1}}{r_{0}^{l+2} r^{l+2}} P_{l}(\cos \theta)
\end{aligned}
$$

где тензоры

$$
\overleftrightarrow{\varepsilon}_{l}^{-1}(\omega \mid \mathbf{B})=\left[l \overleftrightarrow{\varepsilon}_{2}(\omega \mid \mathbf{B})+(l+1) \varepsilon_{3} \overleftrightarrow{\mathbf{I}}\right]^{-1}
$$

взаимно обратны комбинированным тензорам проницаемостей

$$
\overleftrightarrow{\varepsilon}_{l}(\omega \mid \mathbf{B})=\left[l \overleftrightarrow{\varepsilon}_{2}(\omega \mid \mathbf{B})+(l+1) \varepsilon_{3} \overleftrightarrow{\mathbf{I}}\right]
$$

Угловые факторы $P_{l}(\cos \theta)$ представляют собой полиномы Лежандра степени $l$, а $\theta-$ угол между осью $\mathbf{r}_{0}$ и радиусом-вектором, определяющим положение точки, в которой рассчитывается потенциал $\varphi_{j}(r, \theta)$.

Потенциалы $\delta \varphi_{j}(r, \theta \mid \mathbf{B})(j=2,3)$ радиально выстроенного точечного диполя $\mathbf{p}_{0}=q \delta \mathbf{r}_{0}$ КТ получаем дифференцированием (1), (2) по переменной $r_{0}$ :

$$
\delta \varphi_{j}(r, \theta)=\nabla \varphi_{j}(r, \theta) \delta r_{0}
$$




$$
\begin{aligned}
& \delta \varphi_{2}\left(r, \theta ; r_{0} \mid \mathbf{B}\right)=-\sum_{l=1}^{\infty}(2 l+1)(l+1) \mathbf{p}_{0} \overleftrightarrow{\varepsilon}_{l}^{-1}(\omega \mid \mathbf{B}) \mathbf{r} \frac{r^{l-1}}{r_{0}^{l+2}} \\
& \quad \times P_{l}(\cos \theta), r<R_{\mathrm{NP}}, \\
& \delta \varphi_{3}\left(r, \theta ; r_{0} \mid \mathbf{B}\right)=\frac{p_{0}}{\varepsilon_{3} R_{A}^{3}}\left(r \cos \theta-r_{0}\right) \\
& +\frac{1}{\varepsilon_{3}} \sum_{l=1}^{\infty} l(l+1) \mathbf{p}_{0}\left[\overleftrightarrow{\varepsilon}_{2}(\omega \mid \mathbf{B})\right. \\
& \left.-\varepsilon_{e} \overleftrightarrow{I}\right] \overleftrightarrow{\varepsilon_{l}}-1(\omega \mid \mathbf{B}) \mathbf{r} \frac{R_{2}^{2 l+1}}{r_{0}^{l+2} r^{l+2}} P_{l}(\cos \theta), r>R_{\mathrm{NP}} .
\end{aligned}
$$

Заметим, что ближнее поле с потенциалом $\delta \varphi_{3}\left(r, \theta ; r_{0} \mid \mathbf{B}\right) \quad$ (4) определяет безызлучательный перенос энергии экситона КТ вблизи проводящей НЧ к малой частице-акцептору радиусом $R_{A}$ (молекуле или атомно-молекулярному кластеру), находящейся в точке с координатами $(r, \theta)$ в магнитном поле.

В режиме сильного конфайнмента кулоновское взаимодействие между электроном и дыркой мало (но может быть учтено по теории возмущений), а их волновые функции в нулевом приближении могут быть представлены как основные состояния свободных частиц в прямоугольной сферически симметричной потенциальной яме радиуса $R_{c}$ с бесконечно высокими стенками:

$$
\chi_{0}\left(\mathbf{r}_{p}\right)=\frac{1}{\sqrt{2 \pi R_{c}}} \frac{\sin \left(\pi r_{p} / R_{c}\right)}{r_{p}}, \quad p=e, h .
$$

Двухчастичное состояние такого „сжатого экситона“ принимает вид [24]

$$
\psi\left(\mathbf{r}_{e}, \mathbf{r}_{h}\right)=\chi_{0}\left(\mathbf{r}_{e}\right) \chi_{0}\left(\mathbf{r}_{h}\right),
$$

а вектор поляризации КТ

$$
\mathbf{P}\left(r^{\prime}\right)=\frac{\mathbf{d}_{v c}}{2 \pi R_{c}} \frac{\sin ^{2}\left(\pi r^{\prime} / R_{c}\right)}{r^{\prime 2}},
$$

где $d_{v c}-$ векторный матричный элемент межзонного электронного дипольного момента перехода, $r^{\prime}-$ расстояние от центра КТ до точки локализации $e-h$-пары $\left(r^{\prime}=r_{e}=r_{h}\right)$. Дипольный момент $p_{0}$ КТ связан с вектором поляризации соотношением $\mathbf{p}_{0}=4 \pi \int_{0}^{R_{c}} \mathbf{P}\left(r^{\prime}\right) r^{\prime 2} d r^{\prime}$.

Радиальные зависимости электрических потенциалов внутри (3) и вне (4) КТ носят нелинейный характер. С увеличением параметра $r^{\prime}$ до $r^{\prime}=R_{c} / 2$ значение потенциала резко возрастает, а при дальнейшем увеличении $r_{c}$ начинает уменьшаться. Вне КТ потенциал поля постепенно уменьшается с удалением от ее поверхности.

В случае, когда дипольные моменты двух частиц (КТ и НЧ) формируются в неоднородном поле, формула для скорости $w_{s p}(\omega \mid \mathbf{B}, r)$ спонтанного излучения [20] объединенной бинарной системой „КТ-проводящая НЧ“ должна быть модифицирована следующим образом:

$$
\begin{aligned}
& w_{s p}\left(\omega \mid \mathbf{B}, r_{0}\right)=\frac{4}{3} \frac{\omega^{3}}{\hbar c^{3}} \mid \mathbf{p}_{2}\left(r_{0} \mid \mathbf{B}\right)+\frac{3 \varepsilon_{3}}{\varepsilon_{1}+2 \varepsilon_{3}} \\
& \times\left.\int_{0}^{R_{c}} \mathbf{P}\left(r^{\prime}\right) 4 \pi r^{\prime 2} d r^{\prime}\right|^{2}
\end{aligned}
$$

где

$$
\begin{aligned}
\mathbf{p}_{2}\left(r_{0} \mid \mathbf{B}\right) & =-\frac{1}{2 \varepsilon_{3}} \int_{0}^{R_{\mathrm{NP}}} \int_{0}^{\pi}\left[\overleftrightarrow{\varepsilon}_{2}(\omega \mid \mathbf{B})-\varepsilon_{3} \overleftrightarrow{I}\right] \\
& \times \overleftrightarrow{\nabla}_{\mathbf{r}} \delta \varphi_{2}(r, \theta \mid \mathbf{B}) r^{2} d r \sin \theta d \theta .
\end{aligned}
$$

Интеграл $\mathbf{p}_{2}\left(r_{0} \mid \mathbf{B}\right)(6)$ представляет собой наведенный дипольный момент НЧ [25] в неоднородном поле активированной КТ и магнитном поле В.

Аналогичной модификации должно быть подвергнуто выражение для скорости $U\left(\omega \mid \mathbf{B}, r_{0}\right)$ безызлучательной передачи энергии от КТ к НЧ [20]. В случае неоднородного поля эта скорость (без использования величины дипольной поляризуемости) может быть представлена интегралом от мнимой части квадратичной формы вектора напряженности локального поля $\overleftrightarrow{\nabla}_{\mathrm{r}} \delta \varphi_{2}(r, \theta \mid \mathbf{B})=\mathbf{E}_{2}(r, \theta \mid \omega)$ внутри НЧ:

$$
\begin{gathered}
U\left(\omega \mid \mathbf{B}, r_{0}\right)=\frac{1}{2 \pi \hbar} \int_{0}^{R_{\mathrm{NP}}} \int_{0}^{\pi} \operatorname{Im} \mathbf{E}_{2}^{*}(r, \theta \mid \omega) \\
\times \overleftrightarrow{\varepsilon}_{2}(\omega \mid \mathbf{B}) \mathbf{E}_{2}(r, \theta \mid \omega) \sin \theta d \theta r^{2} d r .
\end{gathered}
$$

Ниже для упрощения расчетов вместо выражения (7) использовалась его модифицированная версия в виде интегрального среднего в некотором внутреннем слое радиуса $0<\bar{r}<R_{\mathrm{NP}} \mathrm{HЧ}$ :

$$
U\left(\omega \mid \mathbf{R}, r_{0}\right)=\frac{2 \bar{r}^{3}}{3 \hbar} \operatorname{Im}\left[\mathbf{E}_{2}^{*}(\bar{r} \mid \omega) \overleftrightarrow{\varepsilon}_{2}(\omega \mid \mathbf{B}) \mathbf{E}_{2}(\bar{r} \mid \omega)\right]
$$

Регистрируемой в экспериментах величиной является спектральная плотность $N(\omega \mid \mathbf{B})$ числа фотонов, испущенных объединенной системой „КТ-НЧ““ на частоте $\omega$. Она определяется вероятностью $w_{s p}$ спонтанного перехода (5), умноженной на функцию лоренцевой формы линии с шириной

$$
\Gamma\left(\omega \mid \mathbf{B}, r_{0}, \vartheta\right)=w_{s p}\left(\omega \mid \mathbf{B}, r_{0}\right)+U\left(\omega \mid \mathbf{B}, r_{0}, \vartheta\right)+K
$$

$\left(\vartheta-\right.$ угол между векторами $\mathbf{p}$ и $\left.\mathbf{r}_{0}\right)$,

$$
N\left(\omega \mid \mathbf{B}, r_{0}, \vartheta\right)=\frac{1}{2 \pi} \frac{w_{s p}^{2}\left(\omega \mid \mathbf{B}, r_{0}\right) \Gamma\left(\omega \mid \mathbf{B}, r_{0}, \vartheta\right)}{\left(\omega-\omega_{i f}\right)^{2}+\Gamma^{2}\left(\omega \mid \mathbf{B}, r_{0}, \vartheta\right)}
$$

где $\omega_{i f}-$ резонансная частота лоренцева контура экситонной полосы, $K-$ постоянная скорость одноцентрового безызлучательного распада возбужденной молекулы в отсутствие НЧ. 

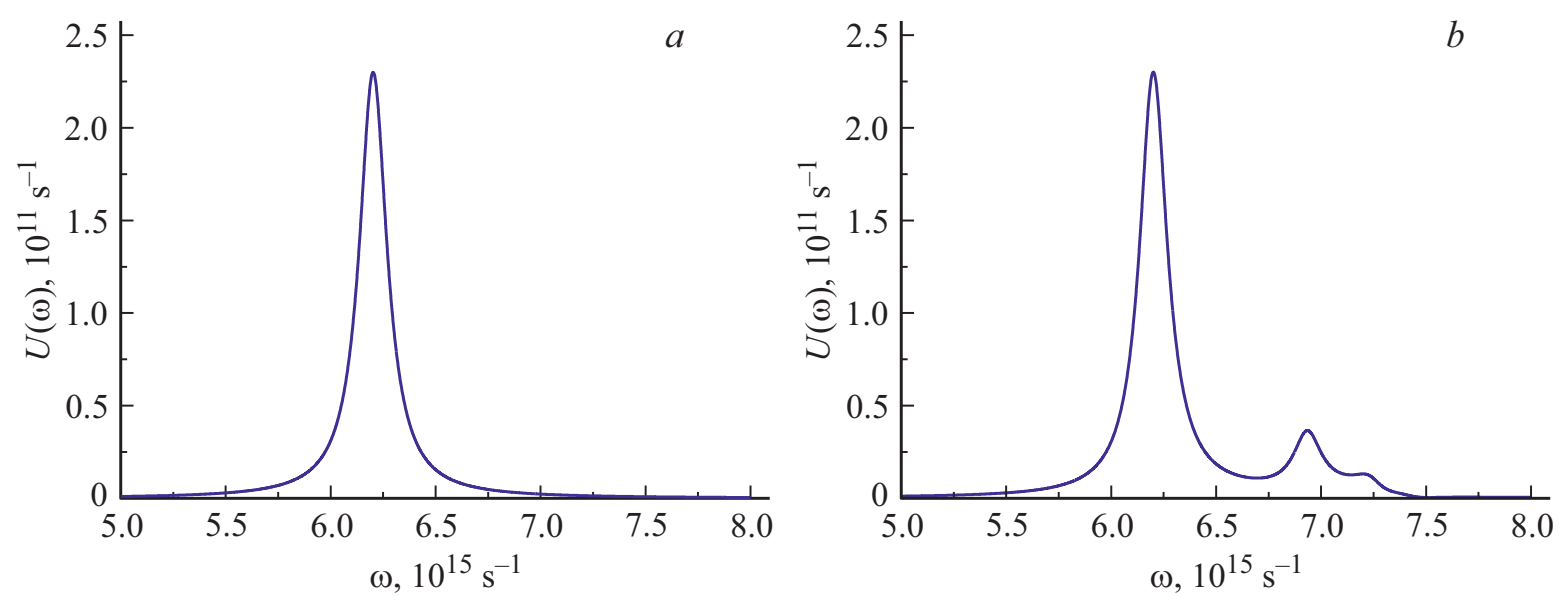

Рис. 2. Спектры скорости безызлучательного переноса энергии от КТ к НЧ при учете нескольких первых мультиполей в отсутствие внешнего магнитного поля: $(a) n=1,(b) n=3 ; \gamma=1.6 \cdot 10^{14} \mathrm{~s}^{-1} ; B=0 \mathrm{~T}$.
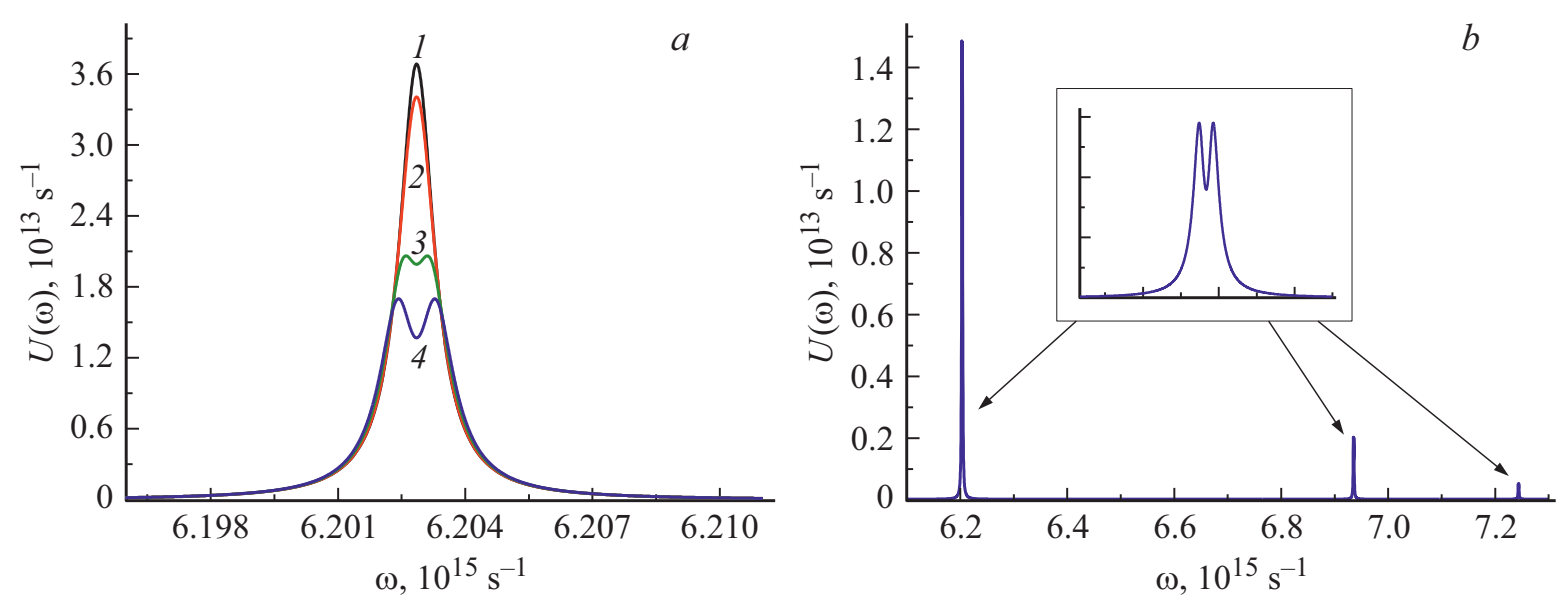

Рис. 3. Спектры скорости безызлучательного переноса энергии от КТ к НЧ в магнитном поле $(a)$ различной индукции $B(1-0$, $2-2,3-6,4-8 \mathrm{~T})$ при учете только мультиполя первого порядка и $(b)$ индукции $B=10$ Т для трех первых мультипольных резонансов. $\gamma=10^{12} \mathrm{~s}^{-1}$.

\section{Результаты исследования}

На основе (3) и (4) были произведены расчеты характеристик поля

$$
\left.-\vec{\nabla}_{\mathbf{r}} \delta \varphi_{j}(r, \theta \mid \mathbf{B})=\mathbf{E}_{j}(r, \theta) \mid \omega\right)
$$

внутри $(j=2)$ и вне $(j=3)$ НЧ. Спектры скоростей излучательного (5) и безызлучательного (7), (8) распадов экситонного состояния комплекса КТ-НЧ, а также спектральная плотность излучаемых им фотонов (9) представлены ниже на рис. 2-8. Для расчетов были использованы несколько различных аппроксимаций обратного тензора

$$
\overleftrightarrow{\varepsilon}_{l}^{-1}(\omega \mid \mathbf{B})=\left[l \overleftrightarrow{\varepsilon}_{2}(\omega \mid \mathbf{B})+(l+1) \varepsilon_{3} \overleftrightarrow{\mathbf{I}}\right]^{-1}
$$

его скалярным аналогом

$$
\overleftrightarrow{\varepsilon}_{l}^{-1}(\omega \mid \mathbf{B}) \rightarrow 1 /\left[l \varepsilon_{2}(\omega \mid \mathbf{B})+(l+1) \varepsilon_{3}\right]
$$

в котором в качестве базового скалярного элемента $\varepsilon_{2}(\omega \mid \mathbf{B})$ использовались различные инварианты разного порядка для тензора $\overleftrightarrow{\varepsilon}_{2}(\omega \mid \mathbf{B})$ : от первого $(1 / 3) \operatorname{Tr} \overleftrightarrow{\varepsilon}_{2}(\omega \mid \mathbf{B})$ - на основе следа, до третьего $\left(\text { Det }\left\|\overleftrightarrow{\varepsilon_{2}}(\omega \mid \mathbf{B})\right\|\right)^{1 / 3}$ - на основе детерминанта. Расчеты производились для всех указанных аппроксимаций, a в итоге были изучены последствия произведенной замены.

Оказалось, что при использовании в качестве базового скалярного элемента $\varepsilon_{2}(\omega \mid \mathbf{B})$ следа $(1 / 3) \operatorname{Tr} \overleftrightarrow{\varepsilon}_{2}(\omega \mid \mathbf{B})$ тензора $\overleftrightarrow{\varepsilon}{ }_{2}(\omega \mid \mathbf{B})$ время расчетов спектров в математическом пакете по формулам (5) и (8), (9) существенно уменьшалось, но при этом давало практически одинаковые результаты с расчетами по точным тензорным формулам. Однако при изучении влияния магнитного поля на радиационные спектры, очевидно, что такая упрощающая расчет замена работать не будет, и необходимо производить расчеты величин только в первоначальном тензорном виде. 
При расчете были использованы следующие постоянные величины, приведенные ниже: $\omega_{p}=$ $=13.87 \cdot 10^{15} \mathrm{~s}^{-1}, \quad B=0 \mathrm{~T}, \quad R_{\mathrm{NP}}=7 \mathrm{~nm}, \quad R_{c}=3 \mathrm{~nm}$, $\Delta=1 \mathrm{~nm}, \quad R_{\mathrm{QD}}=4 \mathrm{~nm}, \quad \bar{r}=3 \mathrm{~nm}, \quad r_{B}=5 \mathrm{~nm}, \quad r_{0}=$ $=R_{\mathrm{NP}}+5 \mathrm{~nm}, \quad d_{v c}=12 \mathrm{D}, \quad \varepsilon_{1}=6, \quad \varepsilon_{3}=2, \quad \theta=\pi / 2$, $K=8 \cdot 10^{13} \mathrm{~s}^{-1}$. Те величины, значения которых изменялись в ходе расчетов, и таким образом отличались от вышеприведенных, указаны в подписях к рисункам. Значения величин, типичных для КТ, взяты из работы [24], а в качестве металла для НЧ использовалось золото.

Для наблюдения изменений в радиационных спектрах бинарной системы КТ-НЧ, индуцированных действием постоянного магнитного поля, необходимо создать условия для высокого разрешения спектральных линий, что может быть обеспечено, если частота столкновений электронов в металле будет меньше на 2-3 порядка, чем при нормальных условиях. В частности, этого можно достичь путем глубокого охлаждения металла до температур менее 100 К [26].

\section{Скорость безызлучательного переноса энергии от KT к Hч}

Спектры скорости $U\left(\omega \mid \mathbf{B}, r_{0}\right)$ (вероятности перехода в единицу времени) безызлучательной передачи энергии от КТ к НЧ в отсутствие магнитного поля и при его наличии были рассчитаны на основе формулы (8). Учет мультиполей различного порядка в формуле (3) для потенциала поля внутри НЧ осуществлялся суммированием по $l$ конечного числа $\mathrm{n}$ членов ряда, т.е. заменой $\sum_{l=1}^{\infty} \rightarrow \sum_{l=1}^{n}$. В отличие от случая однородного поля, когда возбуждается только дипольное колебание, в неоднородном поле КТ отклик системы (НЧ) носит мультипольный характер, что и видно на рис. 2.

На рис. 2, $a$ представлен спектр скорости безызлучательного переноса энергии в дипольном приближении, т. е. при $n=1$. Наблюдается одна спектральная полоса на резонансной частоте $\omega_{1}=6.2 \cdot 10^{15} \mathrm{~s}^{-1}$, определяемая дипольным колебанием зарядов в металлической НЧ. С увеличением числа $n$ до двух кроме основного (дипольного) резонанса в высокочастотной области появляется дополнительная спектральная полоса с максимумом на частоте $\omega_{2}=6.93 \cdot 10^{15} \mathrm{~s}^{-1}$ с меньшей амплитудой, отвечающая квадрупольным колебаниям. При $n=3$ возникает новая полоса на частоте $\omega_{3}=7.24 \cdot 10^{15} \mathrm{~s}^{-1}$ (рис. $2, b)$, которая отвечает октупольным колебаниям, и т.д. Таким образом, спектр скорости безызлучательной передачи энергии от КТ к НЧ в неоднородном электрическом поле донорной частицы представляет собой совокупность мультипольных резонансов на разных частотах, зависящих от порядка мультиполя. Однако основной вклад в суммарную скорость переноса дают два первых резонанса - дипольный и квадрупольный.

На рис. 3 показано, как внешнее магнитное поле изменяет величину скорости переноса и ее резонансную частоту. Из рис. 3, $a$ видно, что спектральная кривая

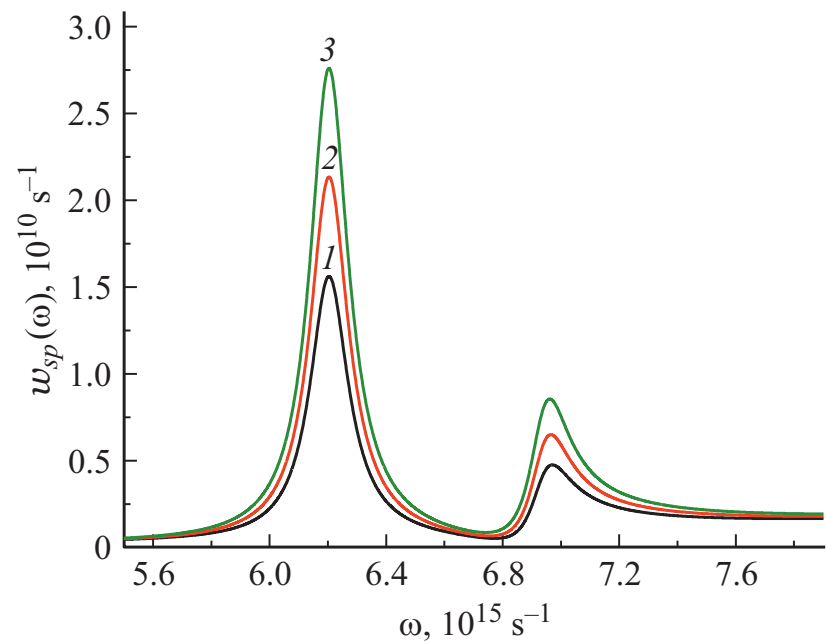

Рис. 4. Зависимость спектра скорости спонтанного излучения системы „КТ-НЧ“ от радиуса НЧ в отсутствие магнитного поля. $n=2, \gamma=1.6 \cdot 10^{14} \mathrm{~s}^{-1}, r_{0}=R_{\mathrm{NP}}+5 \mathrm{~nm}$. Значения $R_{\mathrm{NP}}$ : $1-7,2-8,3-9 \mathrm{~nm}$.

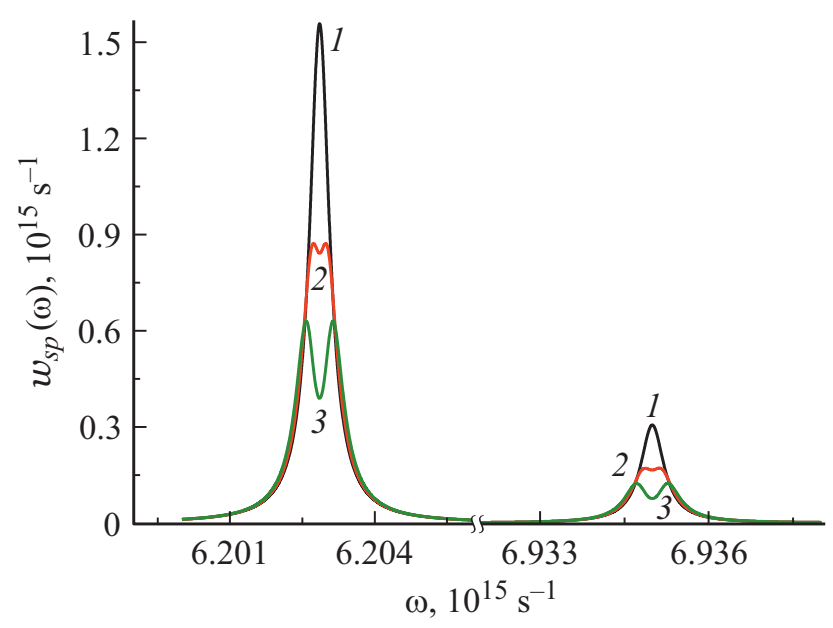

Рис. 5. Зависимость спектра скорости спонтанного излучения системы „КТ-НЧ“ от индукции магнитного поля. $n=2$, $\gamma=5 \cdot 10^{11} \mathrm{~s}^{-1}$. Значения $B: 1-0,2-3,3-5 \mathrm{~T}$.

скорости переноса деформируется в магнитном поле, вначале уменьшаясь по амплитуде (кривая 2), а затем расщепляясь на две компоненты (кривая 3), расстояние между которыми увеличивается с ростом магнитного поля (кривая 4). Все резонансные полосы вне зависимости от того, мультиполю какого порядка они соответствуют, демонстрируют одинаковый магнитополевой отклик (рис. 3, $a$ ): все они расщепляются на две симметричные линии, как это показано на врезке рис. $3, b$.

При увеличении расстояния между КТ и НЧ от 11 до $13 \mathrm{~nm}$ скорость безызлучательного переноса энергии уменьшается на всех резонансных частотах. Очевидно, это связано с тем, что взаимодействие между частицами ослабевает с их удалением друг от друга. 
Напряженность поля внутри НЧ различна в разных ее точках. Из более общей формулы (7) видно, что вклад разных слоев НЧ радиуса $r$ в результирующую скорость переноса будет различной. Чем больше радиус слоя, тем более важную роль в переносе энергии будут играть мультиполи высших порядков. Например, при радиусе НЧ $7 \mathrm{~nm}$ в слое радиусом $6 \mathrm{~nm}$ квадрупольные и октупольные колебания значительно больше по амплитуде, чем те же мультиполи во внутренних слоях меньшего радиуса, и они становятся сравнимыми с дипольными колебаниями.

\section{Скорость спонтанного излучения объединенной системы „КТ-НЧ“}

Эффект влияния металлической НЧ на спонтанные излучательные переходы в КТ при наличии внешнего магнитного поля был исследован на основе выражений (5), (6). В спектрах спонтанного излучения бинарной системой с учетом мультиполей первого и второго порядков присутствуют две соответствующие полосы (рис. 4,5). При учете мультиполя третьего порядка на графике присутствуют все три полосы разной интенсивности.

Как видно из рис. 4, с увеличением радиуса металлической НЧ скорость излучения системы „КТ-НЧ“ увеличивается на обеих резонансных частотах. Как было отмечено ранее, для обнаружения влияния магнитного поля на систему, необходимо уменьшить частоту столкновений электронов $\gamma$ в металле на 2-3 порядка. Увеличение индукции поля от 0 до 5 Т приводит к расщеплению каждой из двух резонансных спектральных полос на две компоненты. Кроме того, с ростом магнитного поля наблюдается уменьшение скорости спонтанного излучения системы (рис. 5), в первую очередь в области резонансных частот.

\section{Спектральная плотность $N(\omega)$ числа фотонов, испущенных объединенной системой „КТ-НЧ“}

Спектральная плотность $N(\omega)$ числа фотонов, испущенных объединенной системой „КТ-НЧ“ на частоте $\omega$, определяется вероятностью $w_{s p}$ спонтанного перехода (5), умноженной на функцию лоренцевой формы линии, с шириной

$$
\Gamma\left(\omega \mid \mathbf{B}, r_{0}, \vartheta\right)=w_{s p}\left(\omega \mid \mathbf{B}, r_{0}\right)+U\left(\omega \mid \mathbf{B}, r_{0}, \vartheta\right)+K,
$$

$\vartheta$ - угол между векторами $\mathbf{p}$ и $\mathbf{r}_{0}$. Функцию $N(\omega)$ можно отождествлять с регистрируемым экспериментально спектром люминесценции объединенной двухчастичной системы. Расчеты таких спектров люминесценции были проведены на основе формулы (9).

На спектрах люминесценции в низкочастотной области наблюдается дополнительная спектральная полоса на резонансной частоте перехода $\omega_{i f}=5.5 \cdot 10^{15} \mathrm{~s}^{-1}$ из возбужденного (экситонного) состояния $i$ КТ в ее основное состояние $f$. Остальные (высокочастотные)

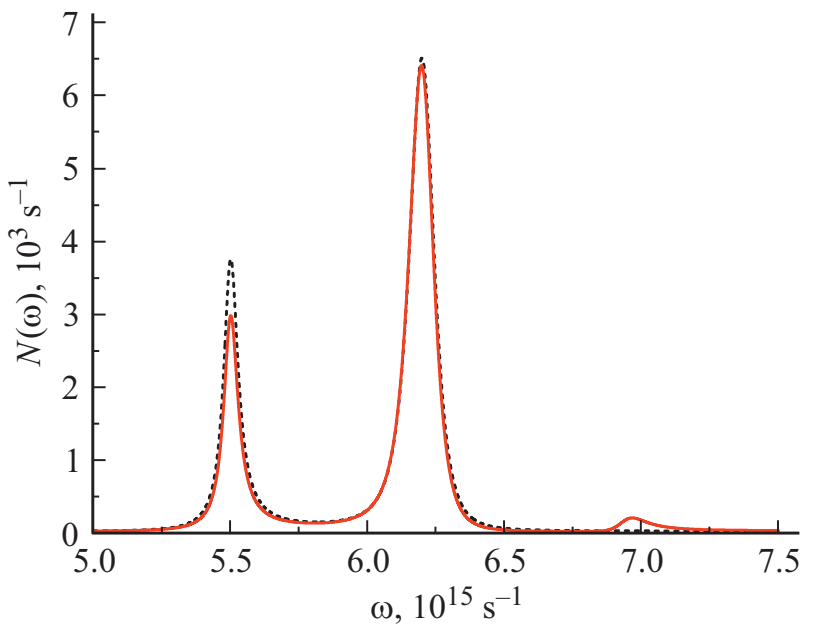

Рис. 6. Спектр люминесценции комплекса „КТ-НЧ“ при разном порядке $n$ мультиполя. $\omega_{i f}=5.5 \cdot 10^{15} \mathrm{~s}^{-1}$, $\gamma=1.6 \cdot 10^{14} \mathrm{~s}^{-1}, K=8 \cdot 10^{13} \mathrm{~s}^{-1}, B=0 \mathrm{~T}$. Пунктирная линия $-n=1$, сплошная $-n=2$.

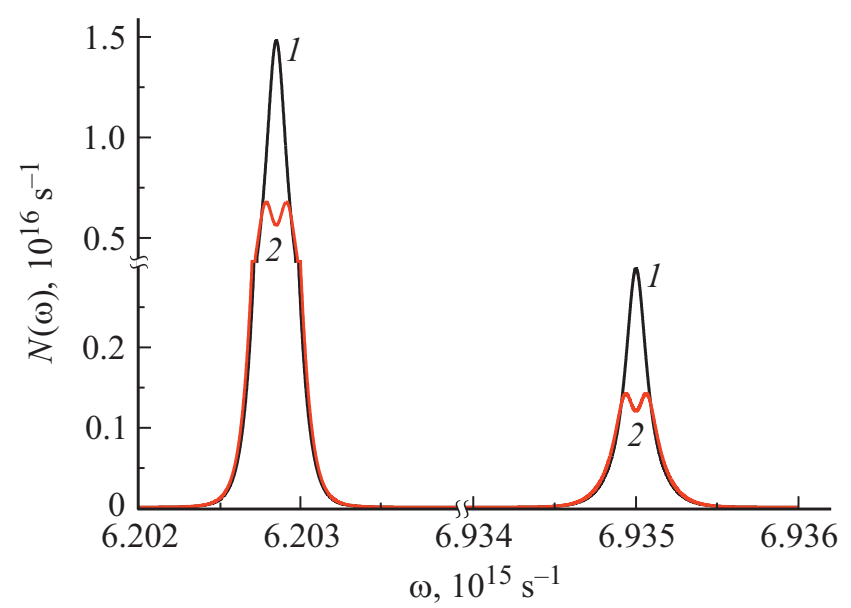

Рис. 7. Спектры люминесценции комплекса „КТ-НЧ“ в отсутствие внешнего магнитного поля (кривая 1) и при его наличии (кривая $2, B=1.2 \mathrm{~T}$ ) в условиях узких плазмонных резонансов при $\gamma=1.6 \cdot 10^{11} \mathrm{~s}^{-1}$. Частота $\omega_{i f}=7 \cdot 10^{15} \mathrm{~s}^{-1}$ совпадает с высокочастотным плазмонным резонансом металлической НЧ. Учтены плазмоны двух низших мультипольностей: $n=1$ и $n=2$.

полосы спектров рис. 6-8 соответствуют плазмонным резонансам металлической НЧ.

На рис. 6 показан график спектральной плотности числа фотонов, испущенных объединенной системой „КТ-НЧ“ на частоте $\omega$ в отсутствие внешнего магнитного поля. Как видно из рисунка, учет мультиполей более высокого порядка способствует перераспределению интенсивности между линиями полного спектра и соответствующему уменьшению люминесценции на резонансной частоте экситонного перехода.

Для демонстрации влияния магнитного поля на спектры люминесценции системы „КТ-НЧ“ частота столк- 


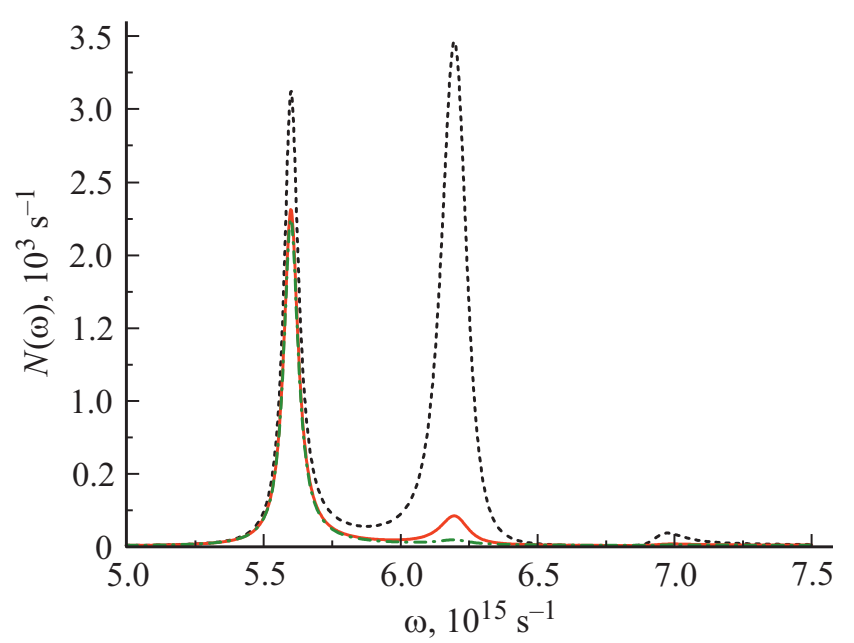

Рис. 8. Зависимость спектра люминесценции объединенного комплекса от расстояния между центрами КТ и НЧ. $n=3$, $\omega_{i f}=5.5 \cdot 10^{15} \mathrm{~s}^{-1}, K=8 \cdot 10^{13} \mathrm{~s}^{-1}$. Значения для $r_{0}$ : пунктирная линия (черная) $-r_{0}=13 \mathrm{~nm}$, сплошная (красная) $r_{0}=17 \mathrm{~nm}$, штрихпунктирная (зеленая) $-r_{0}=21 \mathrm{~nm}$.

новения электронов уменьшалась на 2-3 порядка относительно ее „стандартного“ значения $\gamma=1.6 \cdot 10^{14} \mathrm{~s}^{-1}$, при этом амплитуда резонансных полос резко возрастала, что заметно при сравнении масштабов осей графиков, представленных на рис. 6,7. В последнем случае (рис. 7) при включении магнитного поля заметно расщепление спектральных полос на две компоненты, расходящихся по частотной шкале с увеличением индукции $B$.

При увеличении расстояния между центрами частиц существенно изменяется форма спектров люминесценции: происходит резкое уменьшение амплитуд спектральных полос - плазмонных резонансов, отвечающих вкладу металлической НЧ в суммарный сигнал свечения, и при больших расстояниях $\left(r_{0} \approx 3 R_{\mathrm{NP}}\right)$ основной вклад в люминесценцию дает излучение КТ на частоте $\omega_{\text {if }}$ (рис. 8).

Расчеты показали, что в зависимости от величины диэлектрической проницаемости КТ $\varepsilon_{1}$ происходит ощутимое изменение спектров люминесценции системы. Так, с увеличением $\varepsilon_{1}$ заметно уменьшается люминесценция на частоте экситонного перехода, при этом на частотах плазмонных резонансов это влияние не так значительно. В то же время увеличение межзонного дипольного момента перехода $d_{v c}$ в КТ приводит к увеличению люминесценции на всех резонансных частотах.

Кроме изученных параметрических зависимостей была исследована зависимость люминесценции от степени экситонного конфайнмента посредством замены соответствующего выражения для поляризации КТ в формуле (9). Было установлено, что режим сильного конфайнмента $e-h$ приводит к более интенсивной люминесценции двухчастичного комплекса КТ-НЧ по сравнению со слабым конфайментом практически невоз- мущенного экситона. Как и следовало ожидать, эти отличия особенно хорошо заметны в области частот чисто экситонного перехода в КТ.

\section{Заключение}

На основе специально созданной теоретической модели произведены расчеты частотных зависимостей интенсивности свечения двухкомпонентной системы „экситонактивированная полупроводниковая КТ - плазмонная НЧ“ в постоянном магнитном поле. Исследованы скорости спонтанного излучения системой „КТ-НЧ“ и безызлучательного переноса энергии от КТ к металлической НЧ с учетом неоднородностей ближнего поля КТ. В условиях слабой диссипации наблюдались мультипольные плазмонные резонансы металлической НЧ, испытывающие дублетные расщепления при замагничивании электронной плазмы металла. Было установлено, что с изменением индукции внешнего магнитного поля наблюдается характерная трансформация спектров люминесценции композитной системы в результате экситон-плазмонного взаимодействия составляющих ее компонентов и конкуренции радиационных и безызлучательных каналов распада электронно-возбужденного состояния. Обнаружено, что вне зависимости от мультипольности плазмонных резонансов все они испытывают расщепление во внешнем магнитном поле на две симметричные компоненты, в отличие от экситонной полосы, не являющейся магниточувствительной. Величины расщеплений магниточувствительных полос оказались пропорциональными индукции внешнего поля.

\section{Финансирование работы}

Исследование выполнено при финансовой поддержке Министерства науки и высшего образования Российской Федерации в рамках научного проекта № FSGU-20200003.

\section{Конфлликт интересов}

Авторы заявляют, что у них нет конфликта интересов.

\section{Список литературы}

[1] Bauch M., Toma K., Toma M., Zhang Q., Dostalek J. // Plasmonics. 2014. V. 9. N 4. P. 781. doi 10.1007/s11468-0139660-5

[2] Cao E., Lin W., Sun M., Liang W., Song Y. // Nanophotonics. 2018. V. 7. N 1. P. 145. doi 10.1515/nanoph-2017-0059

[3] Kang L., Chu J., Zhao H., Xu P., Sun M. // J. Mater. Chem. C. 2015. V. 3. N 35. P. 9024. doi 10.1039/C5TC01759A

[4] Yang X., Yua H., Guo X., et al. // Mater Today Energy. 2017. V. 5. P. 72. doi 10.1016/j.mtener.2017.05.005

[5] Jeong N.C., Prasittichai C., Hupp J.T. // Langmuir. 2011. V. 27. N 23. P. 14609. doi 10.1021/la203557f 
[6] Bitton O., Gupta S.N., Haran G. // Nanophotonics. 2019. V. 8. N 4. P. 559. doi 10.1515/nanoph-2018-0218

[7] Balci S., Kocabas C., Küc"uköz B., et al. // Appl. Phys. Lett. 2014. V. 105. N 5. P. 051105. doi $10.1063 / 1.4892360$

[8] Чмерева Т.М., Кучеренко М.Г., Кислов Д.А., Налбандян В.М. // Опт. и спектр. 2018. Т. 125. № 5. C. 670; Chmereva T.M., Kucherenko M.G., Kislov D.A., Nalbandyan V.M. // Opt. Spectrosc. 2018. V. 125. N 5. P. 735. doi 10.1134/S0030400X18110085

[9] Баканов А.Г., Торопов Н.А., Вартанян Т.А. // Опт. и спектр. 2016. Т. 120. № 3. С. 502; Bakanov A.G., Toropov N.A., Vartanyan T.A. // Opt. Spectrosc. 2016. V. 120. N 3. P. 477. doi 10.7868/S0030403416030041

[10] Шамилов Р.Р., Нужоин В.И., Валеев В.Ф. и др. // ЖТФ. 2016. T. 86. № 11. C. 95; Shamilov R.R., Galyametdinov Y.G., Stepanov A.L., et al // Tech. Phys. Russ. J. Appl. Phys. 2016. V. 61. N 11. P. 1698. doi 10.21883/jtf.2016.11.43821.1330

[11] Матюшкин Л.Б., Перцова А., Мошников В.А. // Письма в ЖТФ. 2018. Т. 44. № 8. С. 35; Matyushkin L.B., Pertsova A., Moshnikov V.A. // Techn. Phys. Lett. 2018. V. 44. P. 331. doi 10.21883/PJTF.2018.08.45964.17142

[12] Лебедев В.С., Медведев А.С. // Квант. электрон. 2013. T. 43. № 11. C. 1065; Lebedev V.S., Medvedev A.S. // Quant. Electron. 2013. V. 43. N 11. P. 1065. doi 10.1070/QE2013V043N11ABEH015180

[13] Кучеренко М.Г., Чмерева Т.М. // Опт. и спектр. 2018. T. 125. № 2. C. 165; Kucherenko M.G. Chmereva T.M. // Opt. Spectrosc. 2018. V. 125. N 2. P. 173. doi 10.1134/S0030400X18080179

[14] Чмерева Т.М., Кучеренко М.Г., Курмангалеев К.С. // Опт. и спектр. 2016. Т. 120. № 6. С. 941; Chmereva T.M., Kucherenko M.G. Kurmangaleev K.S. // Opt. Spectrosc. 2016. V. 120 . N 6. P. 881. doi 10.1134/S0030400X16060060

[15] Чмерева Т.М., Кучеренко М.Г. // Изв. вузов. Физика. 2018. T. 61. № 2. C. 91; Chmereva T.M. Kucherenko M.G. // Russ. Phys. J. 2018. V. 61. T. 2. P. 304. doi $10.1007 / \mathrm{s} 11182-018-$ 1402-x

[16] Кучеренко М.Г., Налбандян В.М. // Изв. вузов. Физика. 2016. Т. 59. № 9. С. 87; Kucherenko M.G., Nalbandyan V.M. // Russ. Phys. J. 2017. V. 59. N 9. P. 1425. doi 10.1007/s11182-017-0926-9

[17] Ma Z., Zhang J., Wang X. et al. // Opt. Lett. 2013. V. 38. N 19. P. 3754. doi 10.1364/OL.38.003754

[18] Shikama T., Fujii K., Mizushiri K. et al. // Plasma Phys. and Controlled Fusion. 2009. V. 51. N 2. P. 122001. doi 10.1088/0741-3335/51/12/122001

[19] Кучеренко М.Г., Налбандян В.М. // Опт. журн. 2018. T. 85. № 9. C. 3; Kucherenko M.G. Nalbandyan V.M. // J. Optical Technology. 2018. V. 85. N 9. P. 524. doi 10.1364/JOT.85.000524

[20] Kucherenko M., Nalbandyan V. // Phys. Procedia. 2015. V. 73. P. 136. doi 10.1016/j.phpro.2015.09.134

[21] Briskina C.M., Tarasov A.P., Markushev V.M., Shiryaev M.A. // J. Nanophot. 2018. V. 12. N 4. P. 043506. doi 10.1117/1.JNP.12.043506

[22] Кучеренко М.Г., Налбандян В.М. // Вестник ОГУ. 2015. T. 188. № 13. C. 156.

[23] Гинзбург В.Л., Рухадзе А.А. Волны в магнитоактивной плазме. М.: Наука, 1975. 256 c.

[24] Агранович В.М., Баско Д.М. // Письма в ЖЭТФ. 1999. T. 69. B. 3. C. 232
[25] Климов В.В. Дюклуа М., Летохов В.С. // Квант. электроника. 2001. T. 31. № 7. С. 569; Klimov V.V., Ducloy M., Letokhov V.S. // Quant. Electronics. 2001. V. 31. N 7. P. 569. doi 10.1070/QE2001v031n07ABEH002007

[26] Kucherenko M.G., Nalbandyan V.M. // Eurasian Phys. Tech. J. 2018. V. 15. N 2(30). P. 49. 